Research Letter

\title{
Nasal index of the Tharu and Mongoloid population of Nepal: a cross sectional study
}

\author{
Sarun Koirala, Sandip Shah, Laxman Khanal \\ BP Koirala Institute of Health Sciences, Dharan, Nepal
}

Received 26 June 2014, Accepted 29 July 2014

(C) 2014, Koirala S., Shah S., Khanal L.

(C) 2014, Russian Open Medical Journal

\begin{abstract}
The nasal index measurement is one of the methods anthropologists have used to differentiate living race and subspecies of man. Ethnic influences can result in different appearances of the nose and can be found in many shapes and sizes. A total number of 1000 healthy people (500 males and 500 females) aged between 25-45 years belonging to pure race of Tharu and Mongoloid communities were participated in the study. The nasal index was calculated as (Nasal height / Nasal Breadth) $\times 100$ and their significance was tested by Student t-test. Results - Nasal index among the Mongoloid male and female were 74.6 and 75.9 respectively and the Tharu male and female were 83.8 and 82.4 respectively $(\mathrm{p}<0.05)$. The result of this study revealed significant difference in Nasal index and in Nasal height $(\mathrm{P}<0.05)$ of male and female of Tharu and Mongoloid ethnic races. The study revealed the racial as well as sexual dimorphism pattern in nasal ergonomics in this ethnic group. The sex and ethnicity had considerable effect nasal ergonomics related anthropometric measurements.
\end{abstract}

Keywords: nasal index, ethnicity, anthropometry, dimorphism

Cite as Koirala S, Shah S, Khanal L. Nasal index of the Tharu and Mongoloid population of Nepal: a cross sectional study. Russian Open Medical Journal 2014; 3: 0302.

Correspondence to Dr. Sarun Koirala. Phone: 97798420 53985. Email: poksun@rediffmail.com

\section{Introduction}

The nasal index measurement is one of the methods anthropologists have used to differentiate living race and subspecies of man [1]. Nasal index is defined as ratio of the greatest width of nasal aperture to height of nasal skeleton multiplied by 100 [2]. Based on the index, the nose has been classified into leptorrrhine a fine nosed (69.9), mesorrhine or medium nosed (70.0-84.9) and platyrrhine or broad nosed (85.0) [3]. Environmental climatic conditions also plays a significant role in determining the shape of the nose [4]. The narrower noses are favored in cold and dry climates whereas broader noses in warmer, moister ones as a consequence of natural selection in human evolution [5]. Ethnic influences can result in different appearances of the nose and can be found in many shapes and sizes [6]. Thus nasal index is very useful in anthropology in distinguishing racial and ethnic differences [7]. The nasal index is very useful in anthropology and it is one of the clinical anthropometric parameters recognized in nasal surgical and medical management [8].

Previously, no scientific somatometric data was available for nasal ergonomics, of Tharu and Mongoloid ethnic races to mark the difference. Thus, the aim of this study is to determine the nasal index and to classify the nose type of the pure ethnic races the tharu and mongoloid population living in the Eastern part of Nepal.

\section{Material and Methods}

In the present study, subjects whose parent and grand parent (both maternal and paternal) did not have intercaste marriage were considered as pure race. Rai, Limbu, Magar, Gurung, and Tamang communities were placed in Mongoloid group. Following the Institutional Ethical Review Board clearance, a total number of 1000 healthy people (500 males and 500 females) aged between 25-45 years belonging to pure race of Tharu and Mongoloid communities of Sunsari and Morang district of Eastern Nepal were selected using multistage sampling, snowball technique.

Participant with genetic, endocrine disorders, heart disease, kidney diseases, bony growth, recent nasal surgery, tumor eg. lipoma, sebaceous cyst, visible tumor on head/face etc. were not included. Nasal height (NH) was the distance between Nasion ( $n$ ) and Pronasale (Prn). Nasion ( $n$ ) was the midpoint in fronto- nasal suture and Pronasale (Prn) was prominent point on nose. Nasal Breadth (NB) was the distance between two ala of nose, which is the most laterally placed point on nasal wings. Nasal protusion (sn-prn) was the distance between Subnasale (Sn) and Pronasale (Prn). Subnasale (Sn) was the point which lies at angle between septum and surface of upper lip. Purpose of the study was conveyed and confidentiality and anonymity was assured for each member involved in this study. Considering the convenience of each subject, the personal, present and past histories were taken. All the measurements i.e nasal length, nasal breadth, nasal height were taken by using straight sliding caliper, Martin type anthropometer and head positioned in Frankfurt plane. These are manufactured by Syber Hegne and Company AG, technical products division, Wiesenstrasses $8, \mathrm{CH}-8008$ Zurich, Switzerland. All the collected data were summarized using SPSS 11.5 and the nasal index was calculated as $(\mathrm{NH} / \mathrm{NB}) \times 100$ [9], and their significance was tested by Student t-test. 
Table 1. Nasal length, breadth, height and the Nasal index in the male and female of the Tharu and Mongoloid population

\begin{tabular}{|c|c|c|c|c|}
\hline \multirow[t]{2}{*}{ Parameters } & \multicolumn{2}{|c|}{ Male } & \multicolumn{2}{|c|}{ Female } \\
\hline & Mongoloid & Tharu & Mongoloid & Tharu \\
\hline Nasal height, mm & $47.8 \pm 2.8$ & $44.0 \pm 2.9 *$ & $44.1 \pm 4.9$ & $40.5 \pm 2.3^{*}$ \\
\hline Nasal breadth, $\mathrm{mm}$ & $35.7 \pm 3.0$ & $36.9 \pm 2.5^{*}$ & $33.5 \pm 3.0$ & $33.4 \pm 2.3^{*}$ \\
\hline Protusion, $\mathrm{mm}$ & $12.7 \pm 1.3$ & $11.8 \pm 1.2$ & $11.8 \pm 1.2$ & $11.3 \pm 1.1$ \\
\hline Nasal index & $74.6 \pm 3.1$ & $83.8 \pm 3.0 *$ & $75.9 \pm 5.1$ & $82.4 \pm 2.5 *$ \\
\hline
\end{tabular}

$*$ is $\mathrm{p}<0.05$ between Tharu and Mongoloid population. Data presented as mean and standard deviation (M $\pm S D)$.

\section{Results}

Among male of both ethnic races, Mongoloid male (48.5 \pm 2.8$)$, female $(44.7 \pm 7.0)$ had the highest nasal height, and Tharu male and female had the shortest $(44.0 \pm 2.9,40.5 \pm 2.4$, respectively). There was highly significant difference in nasal height, between male and female of both the races $(p<0.05)$. Tharu male had the broadest nasal breadth (36.9 \pm 2.6$)$ and mongoloid had the least (34.4 \pm 3.7$)$. There was a highly significant difference in nasal height between the male and female in all ethnic races $(P<0.05)$. Mongoloid male had more protruded nose $(12.7 \pm 1.3)$, and Tharus had the least protusion of nose $(11.8 \pm 1.2)(P<0.05)$. Nasal index among the mongoloid male and female was 74.6 and 75.9 respectively, and the Tharu male and female were 83.8 and 82.4 respectively $(p<0.05)$. This is indicative of a sexual dimorphic pattern in the ethnic group studied. The result of this study revealed significant difference $(P<0.05)$ in Nasal index of male/female of Tharu and Mongoloid. For pure ethnic races Tharu and Mongoloid previously no such scientific somatometric data was available nasal ergonomics in this group to mark the difference. This demonstrates the racial as well as sexual differences in nasal ergonomics.

\section{Discussion}

In a study conducted by G.S. Oladipo et al. (2009), in Itsekiri and Urhobo people, the results showed the Urhobos had a mean nasal index of 89.63 and the Itsekiri's had a mean nasal index of $90.74(p<0.05)$, and the two ethnic groups fall within the platyrrhine (short, broad nosed) [10]. The Ekpeye male had the highest mean nasal index of 93.72 and fall under plaryrrhines while the Ikwerre male had lowest mean value of 84.81 and fall under mesorrhine type. Thus racial difference have been reported by several authors $[7,9]$.

In our study, the nasal index of mongoloid male and female was 74.6 and 75.9 and fall under the mesorrhine (medium nose) typ. Similarly the nasal index of the Tharu male and female is 83.8 and 82.4 (wider nose) and also fall under the mesorrhine type but have the broader nose. Male have significantly higher nasal index values than the females in this study. This shows there is the sexual dimorphism. It also indicates the racial and ethnic differences in nasal index amongst different populations. The results are in agreement with R.G. Franciscus and J.C. Long (1991) [7] and G.S. Oladipio (2010) [10], who raported higher values for nasal height, nasal width and nasal index. Similar is the finding in the study conducted by B. Xu et al. (2001) in Jingpo people in China, who have mesorrhine nose type [11].

In a similar study, G.S. Oladipo et al. (2006) reported that the mean values for Nigerian Igbos were 95.9 and 90.8 for male and female respectively. Thus, the Igbos have platyrrhine nose type and also show sexual dimorphism [12]. Our study indicated that the predominant nose type is mesorrhine based on the mean nasal index of 83.8, 82 for male, female respectively in the Tharu with broader nose and mongoloid male and female with 74.6 and 75.9 respectively. This confirm the existence of sexual dimorphism in nasal parameters among the mongoloid and the tharu population living in Nepal.

Nasal index is related to regional and climatic differences [13]. Various studies have indicated racial and ethnic differences in nasal index amongst different populations [12]. Most Caucasians are leptorrhine having long and narrow nose with nasal index of 69.9 or less. The Indo-Aryan is also similar to the Europian, possessing a fine nose [14].

The study conducted by Milgrim showed that there were racial differences in nasal breadth [15]. They found the mean nasal breadth of white female was $31 \mathrm{~mm}$ and South American female $34.4 \mathrm{~mm}$. In this study there was differences in nasal breath between female of two communities. Nasal breadth Tharu female was $33.4 \mathrm{~mm}$ and Mongoloid $33.5 \mathrm{~mm}$ respectively. So, the nose is one of the best clues to racial origin.

The study conducted by L.M. Milgrim et al. (1996) had shown that the mean nasal protusion of Caribbean female was $13.4 \mathrm{~mm}$ which was closer to the mongoloid female was $11.8 \mathrm{~mm}$, while Whites was $19.7 \mathrm{~mm}$ and Central Americans was $19.3 \mathrm{~mm}$, which showed considerable differences [15]. In this present study the mean nasal protusion, ranged from 11.8-11.3 mm among Tharu and 12.7-13.4 mm among Mongoloid. However, nasal protusion of mongoloid female was found to be higher than tharu $(p<0.05)$ in this study. Similar was values seen in Caribbeans $39 \mathrm{~mm}$, Central Americans $39.5 \mathrm{~mm}$ and South Americans $42 \mathrm{~mm}$. The results of this study is similar with our finding that compare anthropometric characteristics of male and females. Most of the authors have concluded the presence of sexual dimorphism in their studied sample.

\section{Conclusion}

The human nose is a dominant feature in facial region which differs in anatomy and morphology among racial groups and of the most characteristic differences during the analysis of ethnic and racial differences. Present study demonstrated that there were racial as well as sexual dimorphism in nasal ergonomics. The sex and ethnicity had considerable effect nasal ergonomics related anthropometric measurements. The mean nasal index of the Tharu and the Mongoloid population has been determined. The Mongoloid and the tharu fall within the mesorrhine nose type but the Tharu have the more wider nose and with more Nasal index near to platyrrhine type. This study provides a normative data of nasal index, which will be a anthropometric tool in differentiating these ethnic groups, forensic medicine, reconstructive surgery and rhinoplastic amongst the ethnic groups under study. 


\section{Acknowledgments}

I would like to express by thanks to the Tharu and Mongoloid communities of Sunsari and Morang district of Eastern Nepal who interestly participated in this study.

\section{Conflict of interest}

There does not exist any conflict of interest in this study.

\section{References}

1. Risely HH. The People of India. 2nd Edn. W.Crooke eds. Calcutta \& Simla: Thacker, Spink \& Co.; London: W. Thacker \& Co., 1915.

2. Porter JP, Olson KL. Analysis of the African American female nose. Plastic Reconstruct Surg 2003; 111(2): 620-626. (PMID: 12560683) (doi: 10.1097/01.PRS.0000042176.18118.99)

3. Willams $P$, Dyson $M$, Dussak JE, Bannister LH, Berry MM, Collins $P$, Ferguson MWJ. Skeletal System. Gray's Anatomy. 3rd Edn. Churchill Livingstone Edinburgh, 1995: 609-612.

4. Last RJ. Anatomy Applied and Regional. 6th Edn. Churchill Livingstone, 1981: 398-403.

5. Hall RL, Hall D.A. Geographic variation of native people along the Pacific Coast. Hum Biol 1995; 67(3): 407-426. (PMID: 7607636)

6. Heidari Z, Khammar T, Khammar M. Anthropometric measurements of external nose in 18-25 year-old Sistani and Baluch aborigine women in the southeast of Iran. Folia Morphol 2009; 68(2): 88-92. (PMID: 19449295

7. Franciscus RG, Long JC. Variation in human nasal height and breadth. Am J Phys Anthropol 1991; 85(4): 419-427. (PMID: 1928315)

8. Hansen B, Mygind N. How often do normal persons sneeze and blow the nose? Rhinol 2002; 40(1): 407-426.

9. Romo T, Abraham MT. The ethnic nose. Facial Plast Surg 2003; 19(3): 296-278. (PMID: 14574634) (doi: 10.1055/s-2003-43162)

10. Oladipo GS, Eroje MA, Fawehinmi HB. Anthropometric comparison of the nasal indices between the Adoni and Okrika ethnis groups of Rivers State, Nigeria. Int J Med Sci 2009; 1(4): 135-137.

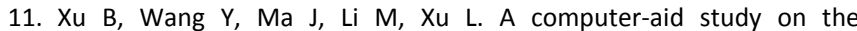
craniofacial features of Archang race in Yunnan province of China. Hua Xi Kou Qiang Yi Xue Za Zhi 2001; 19(6): 394-396. (PMID: 12539693)

12. Oladipio GS, Gwunireama IU, Asawa OD. Anthropometric comparison of nasal inices betweem the Igbos and Yorubas in Nigeria. Glob J Med 2006; 5(1): 37-40. (doi: 10.4314/gjms.v5i1.10147)

13. Farkas LG, Kolar JC, Munro IR. Abstract on the geography of the nose, a morphometric study. Aesthetic Plastic Surg 1986; 10(4): 191-223. (doi: 10.1007/BF01575292) (PMID: 3812136)

14. Sparks CZ, Jantz RL. A reassessment of human cranial plasticity: boas revisited. Proceeding of the National Academy of Sciences 2002; 99: 14636-14639. (doi: 10.1073/pnas.222389599)

15. Milgrim LM, Lawson W, Cohen AF. Anthropometric analysis of the female Latino nose. Revised aesthetic concepts and their surgical implications. Arch Otolaryngeal Head Neck Surgery 1996; 122(10): 1079-1086. (doi:10.1001/archotol.1996.01890220045008) (PMID: 8859120)

\section{Authors:}

Sarun Koirala - MS, Assistant Professor, BP Koirala Institute of Health Sciences, Dharan, Nepal;

Sandip Shah - MS, Assistant Professor, BP Koirala Institute of Health Sciences, Dharan, Nepal;

Laxman Khanal - MS, Resident, BP Koirala Institute of Health Sciences, Dharan, Nepal. 